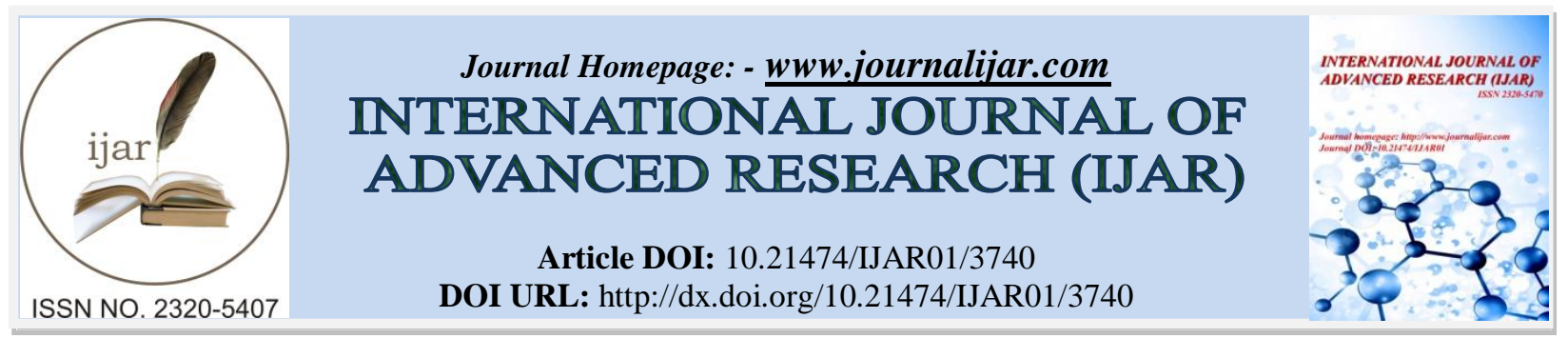

RESEARCH ARTICLE

\title{
PREVALENCE AND ANTIMICROBIAL RESISTANCE PATTERN OF METHICILLIN RESISTANT STAPHYLOCOCCUS AUREUS ISOLATED FROM DIFFERENT CLINICAL SAMPLES.
}

\author{
Dr. Nida Khan ${ }^{1}$, Dr. Vineeta Khare, Dr. Rahmat Farid and Dr. Shadma Yaqoob.
}

\section{Manuscript Info}

Manuscript History

Received: 15 January 2017

Final Accepted: 15 February 2017

Published: March 2017

\begin{abstract}
Background: Methicillin Resistant Staphylococcus aureus (MRSA) since its emergence, has been a challenge for the community owing to its potential to cause life threatening events like sepsis, endocarditis and osteomyelitis.

Aims and objectives: To investigate the prevalence of MRSA in various clinical samples and to find the antibiotic resistance pattern of the MRSA isolates.

Materials and Methods: Oxacillin resistance screening agar with 5.5\% Nacl and Kirby-Bauer disk diffusion method (cefoxitin 30 $\mu \mathrm{g}$ disk) were used for MRSA confirmation and antibiotic resistance testing was done as per CLSI 2016 guidelines. E-test was done for testing sensitivity to Vancomycin.

Result: Staphylococcus aureus was isolated from 350 specimens. Out of these 350 isolates, $220(62.86 \%)$ were methicillin sensitive and rest $130(37.14 \%)$ isolates were MRSA. Most of the MRSA isolates were from urine samples $(43.71 \%)$ followed by pus $(24.0 \%)$, and putum (11.14\%). MRSA isolates showed high resistance to ciprofloxacin $(53.38 \%)$, and clindamycin $(42.31 \%)$. linezolid resistance was seen in only $6.15 \%$ and all isolates were sensitive to vancomycin.

Conclusion: A high prevalence of MRSA (37.14\%.) in our institution warrants the judicious use of antibiotics in treating infections caused by Staphylococcus aureus. Vancomycin and linezolid are good treatment options in infections caused by MRSA isolates.
\end{abstract}

Copy Right, IJAR, 2017,. All rights reserved.

\section{Introduction:-}

The genus Staphylococcus is one of the most commonly encountered group of pathogens in both communityacquired as well as hospital-associated infections. Within genus Staphylococcus, Staphylococcus aureus is the most important and prevalent organism isolated in clinical specimen ${ }^{1}$. The organism also exists as a commensal, colonizing the anterior nares of about one third of the healthy human population. Asymptomatic nasal carriers are at a high risk of subsequent $S$. aureus infection and are presumed to be an important source of strains that spread and cause infection in contacts ${ }^{2,3,4}$.

This centuries-old pathogen is found in almost all the varieties of clinical specimen ranging from minor skin infections to fatal necrotizing pneumonia ${ }^{5}$ and still continues to be the cause of significant morbidity and mortality despite huge advances in medical care $S$. aureus resistance to methicillin was reported for the first time in 1961 in 
England, and since then it continues to be the most dreadful strains of S. aureus. It is one of the most common pathogens that cause nosocomial infections ${ }^{6,7}$

In India, a multicentric trial conducted between January 2008 to December 2009 at several healthcare centres ${ }^{8}$ found MRSA prevalence among specimen collected from outpatients, ward inpatients and ICU to be 28,42 and 43 per cent, respectively in 2008 and 27, 49 and 47 per cent, respectively in 2009, thus indicating that MRSA has assumed a concerning proportion. Moreover, they stressed on the pattern of changing antibiotic susceptibility and also recommended robust antimicrobial stewardship and strengthened infection control measures to prevent spread and reduce emergence of resistance.

It must be kept in mind that current therapeutic options for MRSA are limited few expensive drugs like vancomycin, linezolid, teicoplanin, daptomycin and streptogramins. Another alarming sign is that emergence of resistance to Vancomycin, although at a low level has been reported in literature ${ }^{9}$, thus underlining the observations made by the multicentric study cited above.

Hence, the present study was planned with an aim to assess the prevalence and antibiotic susceptibility of Methicillin resistant Staphylococcus aureus at Era's Lucknow Medical College and Hospital (ELMCH)

\title{
Aims and Objectives:-
}

The present study was carried out with an aim to evaluate the prevalence, antibiotic susceptibility of MRSA in a tertiary care centre. This aim was fulfilled with the help of following objectives:

1. To investigate the prevalence of MRSA in various samples obtained for culture.

2. To find the antibiotic resistant pattern of MRSA isolates

\section{Material And Methods:-}

Place Of Study Study was conducted at Department of Microbiology, Era's Lucknow Medical College.

\section{Duration:}

12 months from Nov 2014 to Oct 2015

\section{Study Population:}

Part I: All patients visiting Era's Lucknow Medical College and Hospital, Lucknow.

\section{Sampling Frame:-}

Sample size: $\quad 350$

\section{Inclusion criteria:-}

All patients including outpatients and admitted patients

\section{Exclusion criteria:-}

Patients who did not show cooperative attitude or refuse to provide necessary information were excluded.

\section{Sample Collection:-}

Part I: The sample collection was done at different clinical Department of Microbiology, Era's Lucknow Medical College, Lucknow using standardized sampling techniques. The specimen obtained from different sources were labeled and tagged with information

\author{
Lab Diagnosis:- \\ Microscopy:- \\ Gram staining \\ Culture was done on following media: \\ Blood agar \\ Mac Conkey agar
}




\section{MRSA confirmation by:-}

Oxacillin resistance screening agar with $5.5 \% \mathrm{NaCl}:-$

Suspend 51.75 grams in $500 \mathrm{ml}$ distilled water. Heat to boiling to dissolve the medium completely. Sterilize by autoclaving at $15 \mathrm{lbs}$ pressure $\left(121^{\circ} \mathrm{C}\right)$ for 15 minutes. Cool at $45-50^{\circ} \mathrm{C}$ and aseptically add rehydrated contents of 1 vial of Oxacillin Resistance Selective Supplement (FD191). Mix well and pour into sterile Petri plates.

Disc diffusion method [Kirby bauer (cefoxitin $30 \mathrm{mg}$ disk)] ${ }^{10}$

Procedure (As per manufactuer's instructions):-

Bacterial Suspension was prepared according to $0.5 \mathrm{McFarland}$ standard and results were interpreted as Resistant, Intermediate or Sensitive for each antimicrobial using CLSI guidelines.

E-TEST:Vancomycin EzyMIC strips ( HiMedia) were used to test for vancomycin sensitivity over a range of MIC from $0.016-256 \mathrm{mcg} / \mathrm{ml}$. The E Test was done and interpreted as per manufacturer's instructions .Apply the Etest strip to the agar surface with the MIC scale facing upwards. MICs were read where the edge of inhibition ellipse intersected the strips.

\section{Statistical Tools Employed:-}

The statistical analysis was done using SPSS (Statistical Package for Social Sciences) Version 15.0 statistical Analysis Software. The values of mean, standard deviation and $\mathrm{p}$ value were calculated and a $\mathrm{p}$ value of $\mathrm{p}<0.05$ was considered significant. Chi square test was done to differentiate between MRSA and MSSA.

\section{Results:-}

A total of 350 specimens from the patients were collected which were subjected to MRSA sensitivity.

Table 1:- Distribution of Cases according to MRSA status $(\mathrm{n}=350)$

\begin{tabular}{|l|l|l|}
\hline & Number of cases & Percentage \\
\hline MRSA & 130 & 37.14 \\
\hline MSSA & 220 & 62.86 \\
\hline
\end{tabular}

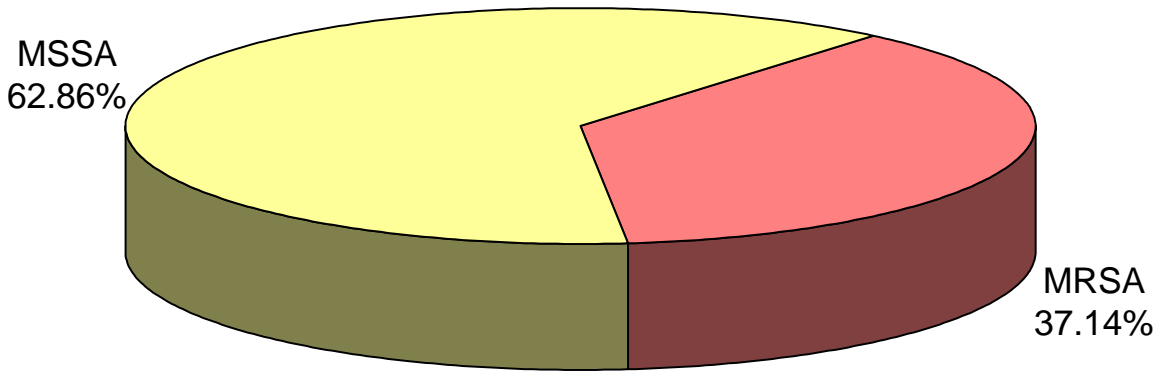

Out of 350 specimens, 220 (62.86\%) were found to be MSSA and only 130 (37.14\%) specimens were found to be MRSA. Prevalence of MRSA in our tertiary care centre was found to be 37.14.

Table 2:- Comparison of Type of specimen between MRSA and MSSA cases

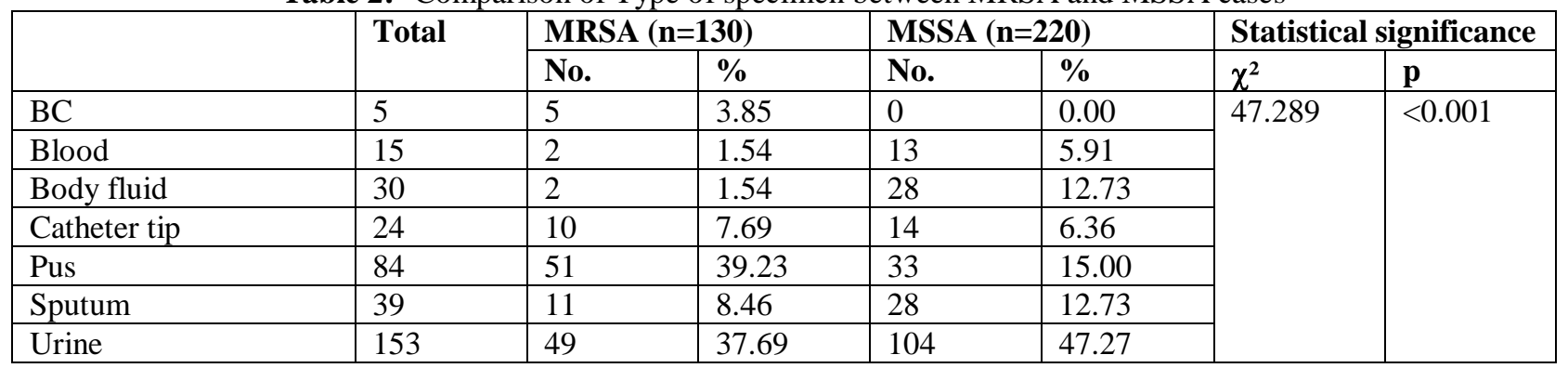


Out of 350 specimens, most common specimen was Urine ( $\mathrm{n}=153 ; 43.71 \%)$, followed by Pus ( $\mathrm{n}=84 ; 24.0 \%)$, Sputum $(n=39 ; 11.14 \%)$, Body fluid $(n=30 ; 8.57 \%) .15(4.29 \%)$ were blood specimens and $5(1.43 \%)$ were from blood culture (BC).

Proportion of MRSA specimens was higher than MSSA for Blood components - Blood culture (3.85\% vs. 0.0\%), Catheter tip (7.69\% vs. $6.36 \%$ ), Pus (39.23\% vs. $15.00 \%)$.

Table 3: Distribution of MRSA positive specimen according to Method of Identification ( $\mathrm{n}=130)$

\begin{tabular}{|l|l|l|l|}
\hline SN & Method & No. of specimen identified & Percentage \\
\hline 1. & Disc diffusion method : Kirby Bauer (cefoxitin 30mg disk) & 130 & 100 \\
\hline 2. & Oxacillin resistance screening agar with 5.5\% NaCl & 90 & 69.2 \\
\hline 3. & Both & 90 & 69.2 \\
\hline
\end{tabular}

All the MRSA samples were identified by Kirby-Baur disc diffusion method (cefoxitin $30 \mathrm{mg}$ ). Using Oxacillin resistance screening agar with 5.5\% NaCl helped in identification of 90 (69.2\%) specimen. Both the methods identified MRSA in $90(69.2 \%)$ specimens

Difference in type of specimens between MRSA and MSSA was found to be statistically significant $(\mathrm{p}<0.001)$.

Table 9:- Comparison of Resistance of MRSA and MSSA cases

\begin{tabular}{|l|l|l|l|l|l|l|}
\hline \multirow{2}{*}{ Resistant } & \multicolumn{2}{l|}{ MRSA $(\mathbf{n}=\mathbf{1 3 0})$} & \multicolumn{2}{l|}{ MSSA (n=22) } & \multicolumn{2}{l|}{ Statistical significance } \\
\cline { 2 - 8 } & No. & $\mathbf{\%}$ & No. & $\mathbf{\%}$ & $\boldsymbol{\chi}^{\mathbf{2}}$ & P \\
\hline CEFOXITIN & 130 & 100.00 & 0 & 0.00 & 350.00 & $<0.001$ \\
\hline CIPROFLOXACIN & 70 & 53.38 & 63 & 28.6 & 22.04 & $<0.001$ \\
\hline DOXYCYCLINE & 20 & 15.38 & 17 & 7.7 & 5.068 & 0.024 \\
\hline NORFLOXACIN & 18 & 13.85 & 15 & 6.8 & 4.726 & 0.030 \\
\hline LINEZOLID & 8 & 6.15 & 8 & 3.6 & 1.187 & 0.276 \\
\hline CLINDAMYCIN & 55 & 42.31 & 47 & 21.4 & 17.37 & $<0.001$ \\
\hline VANCOMYCIN & 0 & 0.00 & 0 & 0 & - & - \\
\hline AMIKACIN & 13 & 10.00 & 11 & 5.0 & 3.198 & 0.074 \\
\hline
\end{tabular}

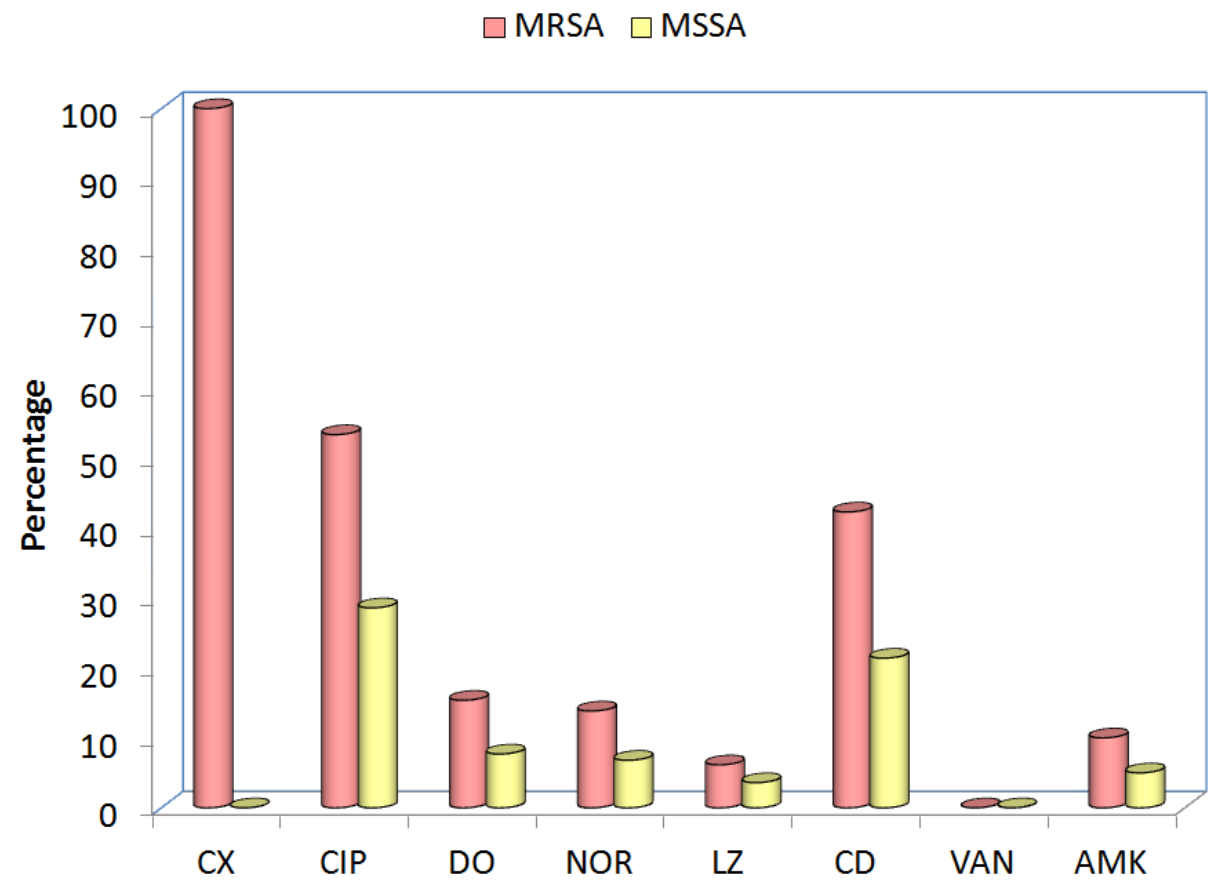


All the MRSA specimen were resistant against Cefoxitin. The resistance rate in decreasing order was Ciprofloxacin (53.38\%), Clindamycin (42.31\%), Doxycycline (15.38\%), Norfloxacin (13.85\%), Amikacin (10\%) and Linezolid $(6.15 \%)$. Vancomycin was sensitive against $100 \%$ of MRSA isolates.

All MSSA specimen were sensitive against Cefoxitin. The resistance rate in decreasing order was Ciprofloxacin (28.6\%), Clindamycin (21.4\%), Doxycycline (7.7\%), Norfloxacin (6.8\%), Amikacin (5\%) and Linezolid (3.6\%). Vancomycin was sensitive against $100 \%$ of MRSA isolates.

\section{Discussion:-}

Methicillin resistant Staphylococcus aureus since its emergence has been a challenge for the healthcare workers owing to its potential to cause life-threatening events like sepsis, endocarditis, and osteomyelitis. Major outbreaks of MRSA and its different phage types have also been recorded and reported in healthcare facilities ${ }^{11}$. Since resistance to multiple antibiotics among MRSA isolates is very common, there is a possibility of extensive outbreaks, which may be difficult to control. MRSA is now one of the commonest nosocomial pathogens, and asymptomatically colonized healthcare workers are the major sources of MRSA in the hospital environment. Early detection of MRSA and formulation of effective antibiotic policy in tertiary care hospitals is of paramount importance from the epidemiological point.

In our study a total of 350 clinical specimen positive for Staphylococcus aureus were obtained and assessed for methicillin resistance. Out of these 130 (37.14\%) were found to be Methicillin resistant. Thus prevalence of Methicillin resistant $S$. aureus was found to be $37.14 \%$ in present study. Prevalence of MRSA has been shown to vary substantially in some contemporary clinical series from the region. Table D1 shows the prevalence of MRSA in some contemporary clinical series:

Table D1:- Prevalence of MRSA in some contemporary clinical series

\begin{tabular}{|l|l|l|l|}
\hline SN & Author (Year) & Sample size and characteristic & MRSA prevalence rate \\
\hline 1. & $\begin{array}{l}\text { Saikia } \text { et al. }(2009)^{12} \text { Dibrugarh, } \\
\text { Assam }\end{array}$ & $\begin{array}{l}276 \\
\text { Clinical specimen }\end{array}$ & 34.78 \\
\hline 2. & $\begin{array}{l}\text { Ahmad } \text { et al. }(2009)^{13} \text {, Armed } \\
\text { Forces Hospital, Saudi Arabia }\end{array}$ & $\begin{array}{l}106 \\
\text { Specimen collected from different hospital }\end{array}$ & $22.3 \%$ \\
\hline 3. & $\begin{array}{l}\text { Tiwari et al. (2011) } \\
\text { Bhubaneswar, India }\end{array}$ & $\begin{array}{l}204 \\
\text { Clinical samples }\end{array}$ & $55.8 \%$ \\
\hline 4. & $\begin{array}{l}\text { Sharma et al. (2013) } \\
\text { Mangalore, India }\end{array}$ & 685 & $23.25 \%$ \\
\hline
\end{tabular}

SAn overview of Table D1 above shows a wide variability in clinical prevalence rate of MRSA in different studies. In present study, Kirby Bauer disc diffusion method (Cefoxitin $30 \mathrm{mg}$ ) proved to be more sensitive than Oxacillin resistance agar method for detection of MRSA. This finding is in accordance with the observations made by Datta $e t$ $a l$. who also showed that Cefoxitin disc diffusion is more sensitive than Oxacillin resitance agar method in the detection of MRSA ${ }^{16}$.

In present study, pus was the most common source of MRSA (39.23\%) followed by urine (37.69\%), sputum $(8.46 \%)$ and catheter tip (7.69\%) respectively. Saikia et al. (2009) ${ }^{12}$ also showed maximum isolation of MRSA from pus/wound swabs $(46.67 \%)$ followed by sputum/throat swab (42.86\%) while Ahmad et al. ${ }^{13}$ found source of MRSA to be $22.2 \%$ from pus, $23.8 \%$ from wound swabs, $33.3 \%$ from aspirates and $13.3 \%$ from sputum. The findings of present study are in accordance with the observations of Tiwari et al. ${ }^{14}$ (2011) who reported pus (45\%) followed by urine $(20.5 \%)$ to be the major source of MRSA in their study. With slight difference in proportions, these two sources comprise the major source of MRSA in our study too. In present study, MRSA positivity rate in pus samples was significantly higher $(51 / 84 ; 60.7 \%)$ as compared to other specimen $(79 / 266 ; 29.7 \%)$. This finding is in accordance with some other studies too that have also reported pus to have a higher MRSA positivity rate as compared to other specimen ${ }^{12,14,15}$.

MRSA specimens were most sensitive against Vancomycin $(100.0 \%)$ and Linezolid $(93.85 \%)$. Cefoxitin, Ciprofloxacin and Clindamycin showed maximum resistance $(100 \%, 53.38 \%$ and $42.31 \%$ respectively). The antibiotic susceptibility has been reported to vary substantially in different studies. Goyal et al(2013) ${ }^{17}$ showed from a study conducted in a teaching hospital at Agra that MRSA specimens were most sensitive against 
Vancomycin,Teicoplanin and Linezolid(100\%),Ampicillin ,Erythromycin and Chloramphenicol showed maximum resistance $\left(100 \%, 88 \%, 66.7 \%\right.$ respectively).Another study conducted by Sharma et al (2013) ${ }^{18}$ from Mangalore ,India showed most of the MRSA strains were sensitive against Linezolid (98.27\%)and Ciprofloxacin (64.7\%) , Tetracycline $(58.38 \%$ ) showed maximum resistance. The finding of the present study are in accordance with Tiwari et al (2011) ${ }^{19}$ Bhubaneswar,India who showed all the isolated MRSA strains were sensitive against Vancomycin and Linezolid(100\%).Vancomycin and Linezolid are most sensitive drugs with most of the studies reporting their sensitivity rates between $90 \%$ to $100 \%$. The findings of present study also emulated the same and showed vancomycin and linezolid to be $100 \%$ and $93.85 \%$ sensitive. For other conventional antibiotics different studies have shown a high resistance pattern. A number of studies have also reported multiresistance. In present study too for conventional antibiotics the resistance rates were ranged from $42.31 \%$ to $100 \%$. A number of specimen were polyresistant too.

These findings in turn indicate the need to develop a antibiotic protocol dependent on MRSA profile of the pathogen in order to reduce the unnecessary burden ofantibiotics.

\section{Conclusions:-}

The present study was carried out in the Department of Microbiology, Era's Lucknow Medical College \& Hospitals to evaluate the prevalence, antibiotic susceptibility and carriage rate of MRSA in a tertiary care centre

1. Out of 350 specimens during the study period, $220(62.86 \%)$ specimens were found to be MSSA and 130 $(37.14 \%)$ were MRSA. Prevalence of MRSA in our institution was $37.14 \%$.

2- Proportion of MRSA specimens was higher than MSSA for Blood culture (3.85\% vs. 0.0\%), Catheter tip (7.69\% vs. $6.36 \%)$, Pus (39.23\% vs. $15.00 \%$ ) while proportion of MSSA was higher than MRSA for body fluid (12.73\% vs. $1.54 \%)$, Sputum $(12.73 \%$ vs. $8.46 \%)$ and Urine (47.27\% vs. $37.69 \%)$.

3-MRSA specimens were most sensitive against Vancomycin (100.0\%) and Linezolid (93.85\%) while least sensitive for Cefoxitin $(0.0 \%)$.

4- 90 isolates were detected by oxacillin resistance screening agar and 130 isolates were detected by Kirby Bauer disk diffusion method.Thus Kirby Bauer method provided an addition of $30.8 \%$ in overall MRSA detection

The findings of present study, thus showed that MRSA was prevalent in our hospital. There is a progressive increase in MRSA positivity and multi-drug resistance in strains of Staphylococci. vancomycin and linezolid were found to have absolute sensitivity.

\section{References:-}

1. Ma X, Ito T, Tiensasitorn C, Jamklang M, Chongtrakool P, Boyle-Vavra S et al. Novel type of staphylococcal cassette chromosome mec identified in community- acquired methicillin resistant Staphylococcus aureus strains. Antimicrobial Agents Chemotherapy, 2002; 46(4): 1147-1152. Link

2. Deleo FR, Otto M, Kreiswirth BN, Chambers HFCommunity-associated

3. methicillin-resistant Staphylococcus aureus. Lancet. 2010; 375: 1557-1568. Link

4. Kennedy AD, Deleo FR. Epidemiology and Virulence of Community-Associated MRSA. Clinical Microbiology Newsletter. 2009; 31: 153-160. Link

5. Kennedy AD, Deleo FR. Epidemiology and Virulence of Community-Associated MRSA. Clinical Microbiology Newsletter. 2009; 31: 153-160. Link

6. Chambers HF, Deleo FR. Waves of resistance: Staphylococcus aureus in the antibiotic era. Nature Reviews Microbiology. 2009; 7: 629-41. Link

7. DeLeo FR, Chambers HF. Re-emergence of antibiotic-resistant Staphylococcus aureus in the genomics era. J Clin Invest., 2009; 119(9): 2464- 2474. Link

8. Jevons MP. “Celbenin”-resistant staphylococci. Br Med J 1961; 124:124- 125. Link

9. 8Jevons MP, Coe AW, Parker MT. Methicillin resistance in staphylococci. Lancet1963;1:904-907. Link

10. Assadullah S, Kakru DK, Thoker MA, Bhat FA, Hussain N, Shah A. Emergence of low level Vancomycin resistance in MRSA. Indian J Med Microbiol 2003;21:196-198. Link

11. National Committee for Clinical Laboratory Standards. 2003. Approved standard: M2-A8. Performance standards for antimicrobial disk susceptibility tests, 8th ed. National Committee for Clinical Laboratory Standards, Wayne, Pa.

12. Cox RA, Conquest C, Mallaghan C, Marples RR. A major outbreak of methicillin resistant Staphylococcus aureus caused by a new phage type (EMRSA-16). J Hosp Infect. 1995;29:87-106. Link 
13. Saikia L, Nath R, Choudhury B, Sarkar M. Prevalence and antimicrobial susceptibility pattern of methicillinresistant Staphylococcus aureus in Assam. Indian Journal of Critical Care Medicine : Peer-reviewed, Official Publication of Indian Society of Critical Care Medicine. 2009;13(3):156-158. Link

14. Ahmad S, Alenzi FQ, Al-Juaid NF, Ahmed S. Prevalence and antibiotic susceptibility pattern of methicillin resistant Staphylococcus aureus at Armed Forces Hospital in Saudi Arabia. Bangladesh Med Res Counc Bull. 2009;35(1):28-30. Link

15. Tiwari S, Sahu M, Rautaraya B, Karuna T, Mishra SR, Bhattacharya S. Prevalence of methicillin-resistant Staphylococcus aureus and its antibiotic susceptibility pattern in a tertiary care hospital. J Indian Med Assoc. 2011;109(11):800-1. Link

16. Sharma NK, Garg R, Baliga S, Bhat K. G. Nosocomial Infections and Drug Susceptibility Patterns in Methicillin Sensitive and Methicillin Resistant Staphylococcus aureus. Journal of Clinical and Diagnostic Research : JCDR. 2013;7(10):2178-2180. Link

17. Datta P, Gulati N, Singla N, Vasdeva HR, Bala K, Chander J, Gupta V. Evaluation of various methods for the detection of meticillin-resistant Staphylococcus aureus strains and susceptibility patterns. J. Med. Microbiol. 2011; 60: 1613-1616. Link

18. Goyal A, Diwakar MK, Bhooshan S, Goyal S, Agrawal A. Prevalence and Antimicrobial SusceptibilityPattern of Methicillin-resistant Staphylococcus aureus [MRSA] isolates at a Tertiary Care Hospital in Agra, North India - A systemic annual review. IOSR Journal of Dental and Medical Sciences (IOSR-JDMS) 2013; 11(6): 80-84. Link.

19. Sharma NK, Garg R, Baliga S, Bhat K. G. Nosocomial Infections and Drug Susceptibility

20. Patterns in Methicillin Sensitive and Methicillin Resistant Staphylococcus aureus. Journal of Clinical and Diagnostic Research : JCDR. 2013;7(10):2178-2180. Link

21. Tiwari S, Sahu M, Rautaraya B, Karuna T, Mishra SR, Bhattacharya S. Prevalence of methicillin-resistant Staphylococcus aureus and its antibiotic susceptibility pattern in a tertiary care hospital. J Indian Med Assoc. 2011;109(11):800-1. Link. 